\title{
THE APPLICATION OF EMBEDDED TECHNOLOGY IN NC SYSTEM
}

\author{
Yong Yin ${ }^{1}$, Zude Zhou', Quan Liu', Fangmin $\mathrm{Li}^{1}$, Yihong Long ${ }^{1}$ \\ 'School of Information Engineering, Wuhan University of Technology; Email: \\ yiyng_hust@126.com
}

Abstract: $\quad$ The paper analyzes the inherent drawbacks of the traditional NC system based IPC, puts forward and then provides the embedded NC system with the architecture of ARM and DSP, in which the ARM takes charge of the G code input, compiling and interpretation, the simulation display, the user's interface and the network interface etc, while the DSP is mainly in charge of real-time tasks such as interpolation computation and path control. The paper also introduces the hardware and software design in detail. Experiments show that $\mathrm{NC}$ system built on the embedded technology can reduce the system hardware scale, facilitate application development, cut down the costs and promote the system's reliability and real-time performance.

Key words: NC system, Embedded technology, ARM, DSP.

\section{INTRODUCTION}

Embedded system is the production integrated with advanced technologies of computer, communication, semiconductor, microelectronics and sensor etc. Its hardware and software can be tailed and configured readily, and it is especially applicable to systems with specific requirements in functionality, reliability, cost, volume and power consumption etc [1].

Present NC systems are mainly based on the IPC (Industrial Personal Computer) architecture, which have their inherent defects on both the structure and the performance [2]. First, the IPC structure is not defined for NC processing. As the IPC is on the basis of the universal structure, it dose not take special account for the NC features from the point of both the bottom hardware design and the operation system environment, thus it is difficult to construct NC system. Second, the NC system of IPC structure

This project is supported by the National Natural Science Foundation of China under the grant No. 50335020.

Please use the following format when citing this chapter:

Yin, Yong, Zhou, Zude, Liu, Quan, Li, Fangmin, Long, Yihong, 2006, in International

Federation for Information Processing (IFIP), Volume 207, Knowledge Enterprise:

Intelligent Strategies In Product Design, Manufacturing, and Management, eds. K. Wang,

Kovacs G., Wozny M., Fang M., (Boston: Springer), pp. 544-549. 
can't ensure the real-time property and reliability. As the IPC with the universal operation system is occupied by many other tasks unrelated with NC processing, which consume large amount of system resource, interfering with the NC's foreground priority. The irrelevant tasks also result into the instability of the NC system. Third, the cost of the NC system on the ground of IPC structure is very high. To equip with the hardware and software of an IPC system will take thousands of dollars, while a piece of embedded microcontroller is no more than fifty dollars, which reduces the cost of the whole system dramatically [3].

In the paper, the application of embedded technology base on ARM7 and DSP to NC system is introduced. The embedded NC system has been developed successfully by Hubei Digital Manufacturing Key Laboratory and Embedded System Research Laboratory in Wuhan University of Technology. The system is based on the S3C44B0 microcontroller of ARM7 series of the Samsung Corporation and the TMS329F2812 microprocessor of the TI Company. The following sections of the paper are to introduce the architecture, the hardware and software design of the system based on embedded technology.

\section{SYSTEM ARCHITECTURE}

The embedded NC system of the paper is not built on IPC, but on the embedded architecture of ARM and DSP. Other accessorial units such as servo control unit, PLC unit, communication unit, display unit and data input unit etc, are also constructed on the basis of embedded technology. The embedded NC system is composed of $\mathrm{G}$ code interpretation module, interpolation module, tools' compensation module, servo control module, operation interface module, network interface module, system management module, simulation and display module and signal detection and fault diagnosis module etc, as shown in Fig 1 . The executive workflow of the system is as follows. The G code interpreter translates the user's codes into instructions to control each motor. The instructions include G, T, S, F and M. For $\mathrm{M}$ instruction, the system sends it to the interpolation module to control the feed motors. The T, M, S and F instructions are accessory, with which the NC system controls the assistant devices such as the cooling liquid controller, the clamps and the lubricant controller etc. As the NC system executes the accessory instructions, it transfers them to the PLC to control the peripheral devices. In the system, the PLC module is designed with softcore, which drastically decreases the cost and reduce the volume of the hardware [4]. Besides executing these accessory instructions, the PLC also accepts the I/O signals from the control panel and the alarm signals from the 
system. The interpolation module, tools' compensation module and the position control module are also the essential unit of the system, whose accuracy and real-time property are the vital indices of the embedded NC system.

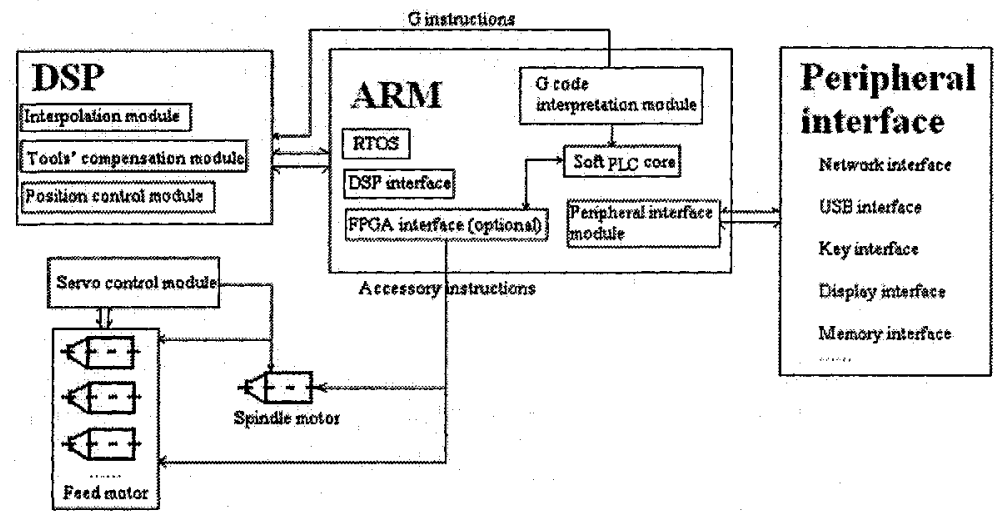

Figure 1. NC system with the architecture of ARM and DSP.

The G code interpretation module, the soft PLC module, the processing simulation module and the operation interface module are implemented in the ARM. So the ARM takes charge of the functions of $G$ code input, compiling and interpretation, the simulation display, the user's interface and the network interface etc. The DSP is mainly in charge of real-time tasks such as interpolation computation and path control.

\section{SYSTEM HARDWARE}

The main control CPU of the system is S3C44B0 chip. This chip has abundant and powerful peripheral resources and communication ability with very high operation and data processing speed, so it can realize functions of data collection, disposing and process control conveniently. The circuit board is four layers with $5 \mathrm{v}$ DC power support and its highest main system frequency is $66 \mathrm{MHz}$. The system is configured with $32 \mathrm{M}$ Flash, $16 \mathrm{M}$ SDRAM, two USB interface of USB2.0 protocol, two Ethernet interface with RTL8019AS chip, one 256 color STN LCD with $640 * 480$ pixels and one PS2 keyboard interface, as shown in Fig2

As there is no control and driving core of CAN bus protocol inside S3C44B0, CAN bus controller and driver are indispensable in the system. In here we choose SJA1000CAN CAN bus controller and 82C250 bus driver of Philips Company to implement the CAN bus interface with S3C44B0 chip, as shown in Fig3. 


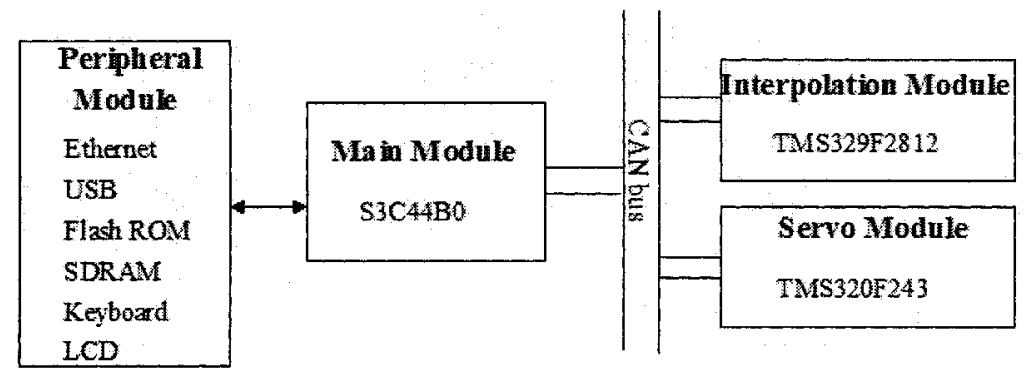

Figure2. System hardware framework

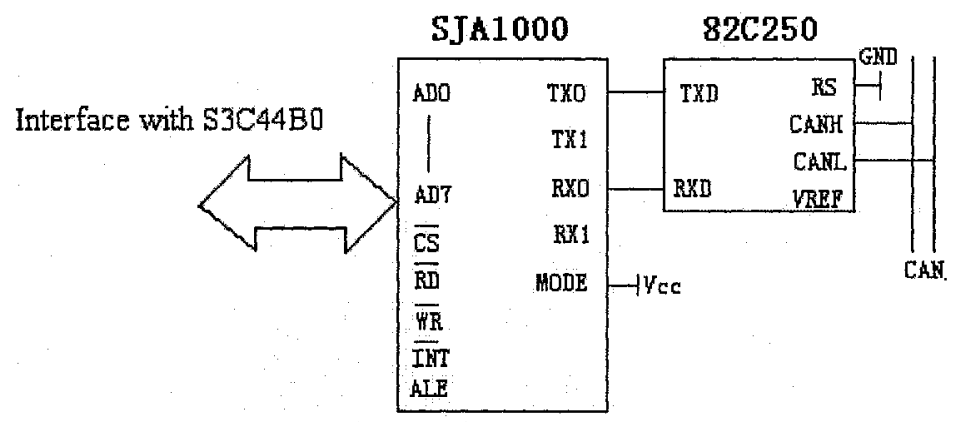

Figure 3. CAN bus interface with S3C44B0

Real-time tasks such as interpolation computation and path control are in the charge of DSP. In the system, the DSP is adopted of TMS329F2812 microprocessor of the TI Company, with its highest main frequency $150 \mathrm{MHz}$ and its clock cycle $6.67 \mathrm{~ns}$, which meets the requirement of the system's real-time performance absolutely. The chip TMS329F2812 microprocessor communicates with $\mathrm{S} 3 \mathrm{C} 44 \mathrm{~B} 0$ chip through $\mathrm{CAN}$ bus. As a CAN bus controller is integrated into TMS329F2812 microprocessor, that is, all functions of the data link layer and application layer of CAN bus protocol is realized inside the TMS320F 2812 chip, only a CAN bus drivers $82 \mathrm{C} 250$ is needed in the system to implement CAN bus linkage.

As DC motor, AC motor or step motor can all be used in the NC system, considering the generality and reconfiguration of the system, the servo module is also linked to the system through CAN bus. Since a clock frequency of $20 \mathrm{MHz}$ is enough for servo control, taking cost into account, the TMS320F243 chip of TI Company is adopted as servo CPU, not TMS329F2812 chip. TMS320F243 is one of the signal processing chips of the Fixed-point series, which is designed specifically for digital motor control. Because a CAN bus controller is also integrated into TMS320F243 chip, it is easy to fulfill communication functions through CAN bus between 
the main control module of S3C44B0 and the servo module of TMS320F243 and to achieve the reconfiguration functions of the system.

\section{SYSTEM SOFTWARE}

The software architecture of the embedded NC system can be separated into three parts, namely the operation system environment, embedded NC core and application program.

The RTOS in the embedded NC system is based on U-cosii, which is transplanted into the ARM microcontroller, providing file system, network interface, memory management and multi-task schedule etc to the NC system. The embedded NC core software, including the PLC unit, I/O control unit, position control unit, G code interpretation unit and tool's compensation unit etc, are supplied in the form of API to the user's application program. On the upper layer, users can develop various kinds of application program according to specific numerical controlled processing.

As U-cosii is a high performance, deterministic, real-time, scaleable, reliable and modularized RTOS, it can be embedded into the NC system based on ARM readily, and users can extend or delete functional modules of both hardware and software easily [5].

In the system, closed-loop adaptive motion control is introduced, so the tool path can be adjusted adaptively in a pre-specified manner with the online feedback from the processing. A fuzzy-PID control algorithm is implemented in DSP, which improves the control accuracy of the system. The system adopts a two-step interpolation algorithm, namely first rough interpolation and second fine interpolation. The first step is in the charge of ARM processor, which outputs not control pulses, but many line sections to the DSP processor. DSP takes charge of the second step, namely it marks out the motion paths of each feed axis, and then outputs fine and real-time motion control pulses.

\section{CONCLUSIONS AND FUTURE WORK}

An economical embedded NC system prototype shown in Fig4 has been developed successfully in the Embedded Lab of Wuhan University of Technology. 


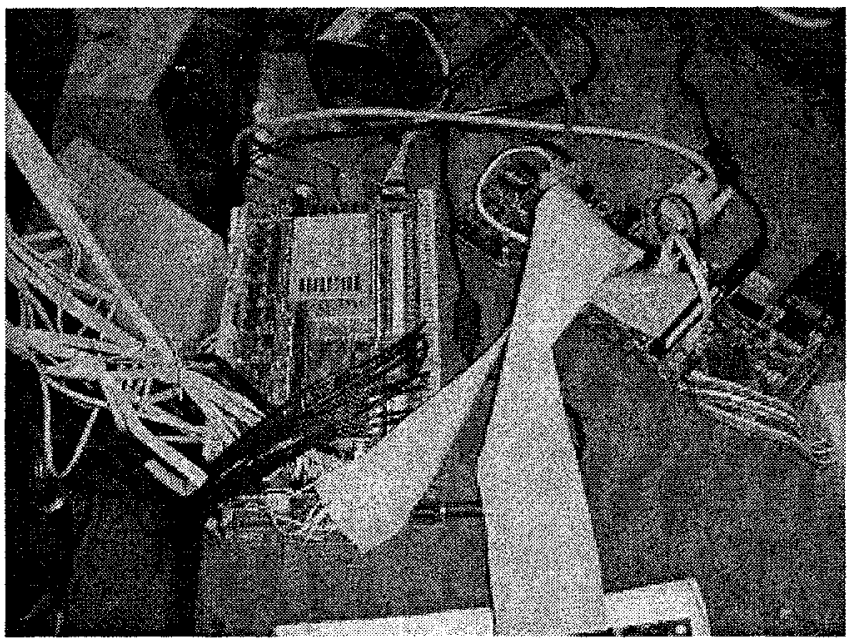

Figure4. Fig6 Embedded NC system prototype

Experiments over the prototype demonstrate that $\mathrm{NC}$ system built on the embedded technology can reduce the system hardware scale, facilitate application development, cut down the cost and enhance the system's reliability and real-time performance. Members in the embedded lab of WHUT are bending themselves to realize core NC system functions on SOC (System On Chip) and try to realize user interface based on MiniGUI. We hope that products based on this technology have a perspective market in the future.

\section{REFERENCES}

1. Zhang X, (2003), Embedded System and Embedded Operation System, Modern Electronic Technique, vol.8, pp.20-24

2. Zhou. Z.D, Liu, Q, Long. Y.H, Li. F.M, (2005), Embedded Technology \& Digital Manufacturing. Digital Manufacturing Science, vol.3. pp.1-10

3. Sun. K, Wang. T.M, Wei. H.X, (2003), Design and Implement of Embedded and Reconfigurable CNC System Based on ARM, Machine Tool \& Hydraulics, vol.6, pp.302304

4. Zhou. Z.D, Chen. Y.P, Yu. W.Y, (2001) Concept and Related Scientific Problems of Digital Manufacturing. China Mechanical Engineering, vol.12, pp.100-104

5. Han. Q.K, Deng. Q.X, Wen. B.C, (2005), Applications of Embedded Technology to Large Intelligent Equipmennts. Digital Manufacturing Science, vol.3, pp.1-17 